\title{
Chrysin protects against renal ischemia reperfusion induced tubular cell apoptosis and inflammation in mice
}

\author{
MINGWEI XU, HONGBO SHI and DONGCAO LIU \\ Department of Urology, Xiangyang Central Hospital, Affiliated Hospital of Hubei \\ University of Arts and Science, Xiangyang, Hubei 441021, P.R. China
}

Received April 24, 2018; Accepted December 17, 2018

DOI: $10.3892 /$ etm.2019.7189

\begin{abstract}
Renal ischemia reperfusion (IR) is a major cause of acute kidney injury with no effective treatment. Chrysin is an anti-inflammatory, anti-oxidant and anti-cancer agent. However, the effect of chrysin on renal IR injury remains unknown. In this study, sham operation, IR and IR+chrysin group mice were treated with or without renal IR injury. For renal IR, bilateral renal pedicles were clamped for $30 \mathrm{~min}$ and then released for $48 \mathrm{~h}$ of reperfusion. Blood and kidney samples were collected for analysis. Results demonstrated that chrysin pretreatment remarkably decreased the levels of serum creatinine and blood urea nitrogen and attenuated morphological abnormalities in renal IR injury. Consistently, tubular cell apoptosis and inflammation were more attenuated in the chrysin pretreatment group compared with the IR group. Chrysin pretreatment decreased the expression of Bax and cleaved caspase- 3 and increased the expression of Bcl-2 in renal IR injury. Furthermore, chrysin administration decreased the mRNA and protein levels of tumor necrosis factor- $\alpha$, interleukin (IL)-1 $\beta$, and IL-6. Furthermore, the I $\kappa \alpha /$ nuclear factor- $\kappa \mathrm{B}$ signaling pathway was more suppressed in the chrysin pretreatment group compared with the IR group. In conclusion, chrysin protects against tubular cell apoptosis and inflammation in renal IR injury.
\end{abstract}

\section{Introduction}

Renal ischemia reperfusion (IR) injury is a common cause of acute kidney injury (AKI) (1) and characterized by high morbidity and mortality (2). In clinical settings, patients subjected to kidney transplantation and renal tumor resection inevitably suffer from renal IR injury (3). Renal tubular cell

Correspondence to: Dr Dongcao Liu, Department of Urology, Xiangyang Central Hospital, Affiliated Hospital of Hubei University of Arts and Science, 136 Jingzhou Street, Xiangyang, Hubei 441021, P.R. China

E-mail: liudongcao1984@163.com

Key words: renal, ischemia reperfusion, chrysin, apoptosis, inflammation apoptosis and inflammatory response are the most important pathophysiological process of ischemic AKI (4). Following IR, the tubular cells in the outer medulla suffer the most severe injury, leading to renal dysfunctions (5). In addition, inflammatory response promotes renal dysfunctions and progressive chronic kidney disease (6). Therefore, inhibiting tubular cell apoptosis and inflammatory response may be an effective treatment of renal IR injury.

Chrysin is a naturally occurring flavonoid with anti-inflammatory, anti-oxidant and anti-cancer properties (7). It ameliorates indomethacin-induced inflammatory response and oxidative injury (8), and suppresses tumor growth of murine melanoma (9). Additionally, it attenuates focal cerebral IR injury in mice (10). However, its effect on renal IR injury remains unknown.

In this study, a renal IR injury model was established in mice and the effects of chrysin on renal IR injury were investigated. Results demonstrated that chrysin remarkably attenuated IR-induced renal dysfunctions and morphological abnormalities. Furthermore, chrysin inhibited renal IR-induced tubular cell apoptosis and inflammatory response. Therefore, chrysin may protect against renal IR-induced ischemic AKI.

\section{Materials and methods}

Animals and treatment. All experiments were approved by the Institutional Animal Care and Use Committee at Hubei University of Arts and Science (Xiangyang, China). The surgical procedures were performed in accordance with the National Institutes of Health Guide for the Care and Use of Laboratory Animals (NIH Publications no. 8023, revised 1978). A total of 30 male C57BL/6 mice (8-10 weeks old) were purchased from the Center of Experimental Animals of Wuhan University (Wuhan, China) and housed in a humidity (50-60\%) and temperature-controlled environment with a 12-h light/dark cycle and free access to food and water. Mice were randomly divided into three groups (each, $n=10$ ): Sham, IR and IR+chrysin. To induce renal IR injury in vivo, the mice were abdominally anesthetized with phenobarbital sodium (60 $\mathrm{mg} / \mathrm{kg}$ ) and their body temperature was maintained at $37^{\circ} \mathrm{C}$. Flank incisions were also conducted to expose the pedicels. The IR and IR+chrysin group mice were subjected to bilateral renal pedicel clamping for $30 \mathrm{~min}$ and reperfusion for $48 \mathrm{~h}$. The sham group mice only underwent exposed pedicles 
without pedicle clamping and received injections of an equal volume of saline. Blood and kidney samples were collected for analysis. Chrysin was purchased from Sigma-Aldrich; Merck KGaA, Darmstadt, Germany (95082) and IR+chrysin group mice were injected with chrysin for 3 days $(100 \mathrm{mg} / \mathrm{kg}$ each time) prior to IR operation.

Renal function assay. The blood $(200 \mu \mathrm{l})$ was collected and centrifugal $(3,500 \mathrm{x} \mathrm{g})$ at $4^{\circ} \mathrm{C}$ for $15 \mathrm{~min}$. Thereafter, the supernatant was harvested and stored at $-80^{\circ} \mathrm{C}$. The serum concentrations of creatinine $(\mathrm{Cr})$ and blood urea nitrogen (BUN) were tested using creatinine and urea assay kits (Nanjing Jiancheng Bioengineering Research Institute, Nanjing, China) in accordance with the manufacturer's protocol.

Hematoxylin and eosin $(H \& E)$ assay. To evaluate kidney injury score, renal samples were fixed in $4 \%$ formaldehyde at room temperature for $24 \mathrm{~h}$, embedded in paraffin and cut into $4 \mu \mathrm{m}$ sections, stained with hematoxylin (8 $\mathrm{min})$, and eosin (2 min) at room temperature. Histological features were imaged using a light microscope (Olympus Corporation, Tokyo, Japan) and a total of 10 random fields of view were obtained from the cortico-medullary region. For assessment of renal injury score, tubular apoptosis, cellular casts and tubular injury were included. The scoring was as follows: $0(<10 \%), 1(10-25 \%)$, $2(25-50 \%), 3(50-75 \%)$ and $4(>75 \%)$. The percentage of renal injury was quantified and conducted in a blinded manner.

Terminal deoxynucleotidyl transferase dUTP nick-end labeling (TUNEL) assay. TUNEL is the method of using the TdT enzyme to covalently attach a tagged form of dUTP to 3 'ends of double- and single-stranded DNA breaks in cells. It is a reliable and useful method to detect DNA damage and cell death in situ. In the present study, renal samples were collected and fixed in $4 \%$ paraformaldehyde at room temperature for $24 \mathrm{~h}$, paraffin embedded, and sectioned at $4 \mu \mathrm{m}$. Then the slides were deparaffinized, rehydrated and the antigen was exposed. The death of tubular cells was detected with Cell Death Detection kit (Fluorescein; Roche Diagnostics, Indianapolis, IN, USA) in accordance with the manufacturer's protocol. Incubated with the tunnel reagent at $37^{\circ} \mathrm{C}$ for $2 \mathrm{~h}$ and mounted with antifade. Image Pro-Plus software version 6.0 (Media Cybernetics, Inc., Rockville, MD, USA) was used for positive cell counting.

Immunohistochemistry (IHC) assay. Renal samples were collected and fixed in $4 \%$ paraformaldehyde at room temperature for $24 \mathrm{~h}$, paraffin embedded, and sectioned at $4 \mu \mathrm{m}$. Then, the slides were deparaffinized, rehydrated in a descending alcohol series and the antigen was exposed by 20 min of incubation at $100^{\circ} \mathrm{C}$ in sodium citrate. Thereafter, the slides were incubated with $3 \% \mathrm{H}_{2} \mathrm{O}_{2}$ at $37^{\circ} \mathrm{C}$ for $10 \mathrm{~min}$ to block the endogenous peroxidase activity. Sequentially, the slides were incubated with bovine serum albumin (cat. no. abs957; Absin Bioscience Inc., Shanghai, China) and the primary antibodies (cleaved Caspase 3, cat. no. 9664, 1:200, Cell Signaling Technology, Inc., Danvers, MA, USA; MPO, cat. no. abs120616, 1:200, Absin Bioscience Inc.) overnight at $4^{\circ} \mathrm{C}$. After incubation with biotin-labeled secondary antibody (abs957, Absin Bioscience Inc.) at room temperature for
Table I. Primers for qPCR detection.

\begin{tabular}{ll} 
Gene & \multicolumn{1}{c}{ Sequence 5'-3' } \\
\hline$\beta$-actin & F: GTGACGTTGACATCCGTAAAGA \\
& R: GCCGGACTCATCGTACTCC \\
IL-1 $\beta$ & F: CCGTGGACCTTCCAGGATGA \\
& R: GGGAACGTCACACACCAGCA \\
IL-6 & F: AGTTGCCTTCTTGGGACTGA \\
& R: TCCACGATTTCCCAGAGAAC \\
TNF- $\alpha$ & F:CATCTTCTCAAAATTCGAGTGACAA \\
& R: TGGGAGTAGACAAGGTACAACCC
\end{tabular}

IL, interleukin; TNF, tumor necrosis factor.

10 min, the color was developed with 3,3'-diaminobenzidine at room temperature for $30 \mathrm{sec}$. Then, the tissues were imaged by fluorescence microscopy (Olympus Corporation). The staining was evaluated in a blinded manner.

Reverse transcription-quantitative polymerase chain reaction (RT-qPCR) assay. Total RNA was isolated using TRIzol reagent (\#15596-026; Invitrogen; Thermo Fisher Scientific, Inc., Waltham, MA, USA) and then reverse-transcribed into cDNA by using a Transcriptor First-Strand cDNA Synthesis kit (\#04896866001; Roche) in accordance with the manufacturer's protocol. SYBR Green (\#04887352001; Roche) was used to quantify the PCR-amplification products. qPCR was performed under the following conditions: Initial denaturation at $95^{\circ} \mathrm{C}$ for $30 \mathrm{sec}$, followed by 40 cycles of $5 \mathrm{sec}$ at $95^{\circ} \mathrm{C}$, $30 \mathrm{sec}$ at $60^{\circ} \mathrm{C}$, and $60 \mathrm{sec}$ at $72^{\circ} \mathrm{C}$. $\beta$-actin was used as the reference gene. The threshold cycle values of the samples were measured using the $2^{-\Delta \Delta C q}$ data analysis method (11). The results were presented as mean \pm standard deviation and SPSS software version 19.0 (IBM Corp., Armonk, NY, USA) was used in the analysis. The primer pairs used in this study are listed in Table I.

ELISA. The blood was collected and centrifuged $(3,500 \mathrm{x} \mathrm{g})$ at $4^{\circ} \mathrm{C}$ for $15 \mathrm{~min}$. Thereafter, the supernatant was harvested and stored at $-20^{\circ} \mathrm{C}$. The protein levels of TNF- $\alpha$ (cat. no. EK0527), IL-1 $\beta$ (cat. no. EK0394) and IL-6 (cat. no. EK0411) were detected by ELISA kits (Boster Biological Technology, Wuhan, China) at $450 \mathrm{~nm}$ in accordance with the manufacturer's protocol.

Western blot analysis. Total protein (kidney) was isolated using radioimmunoprcipitation lysis buffer (cat. no. P0013B; Beyotime Institute of Biotechnology, Haimen, China) and the concentration was detected with bicinchoninic acid reagent (cat. no. \#23225, Thermo Fisher Scientific, inc.). Equal amounts of proteins $(30 \mu \mathrm{g})$ were loaded, proteins were separated with $10 \%$ SDS-PAGE gels and then transferred onto polyvinylidene difluoride membranes (EMD Millipore, Billerica, MA, USA). Thereafter, such proteins were blocked in 5\% skim milk for $1 \mathrm{~h}$ and incubated overnight at $4^{\circ} \mathrm{C}$ with primary antibodies

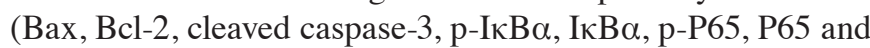
GAPDH). Following incubation in secondary antibodies (cat. 
A

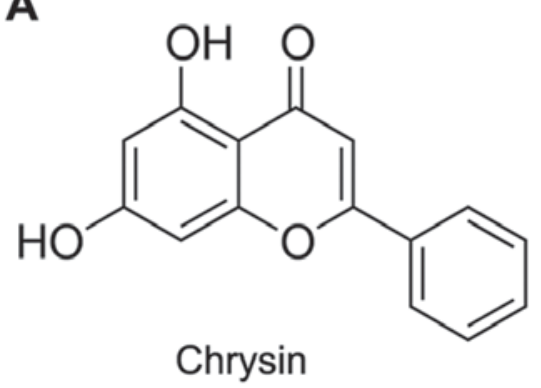

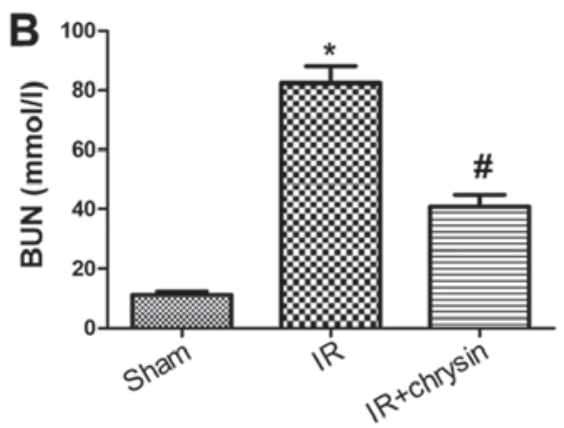

Figure 1. Chrysin pretreatment attenuates IR-induced renal dysfunctions. (A) The chemical structure of chrysin. The serum levels of (B) BUN and (C) creatinine in the sham, IR, and IR+chrysin group. ${ }^{*} \mathrm{P}<0.05$ vs. the sham group; ${ }^{\#} \mathrm{P}<0.05$ vs. the IR group. IR, ischemia reperfusion; BUN, blood urea nitrogen.

no. 926-32211; IRDye 800CW goat anti-rabbit IgG $(\mathrm{H}+\mathrm{L})$; LI-COR Biosciences, Lincoln, NE, USA) for $1 \mathrm{~h}$ at room temperature, washed three times and the membranes were scanned with the Odyssey infrared imaging system (LI-COR Biosciences).

Antibodies. The antibodies against Bax (cat. no. ab32503), Bcl-2 (cat. no. ab32124) and GAPDH (cat. no. ab181602) were obtained from Abcam and used in western blotting. The antibodies against cleaved caspase-3 (cat. no. 9661; used in IHC and western blotting) and phosphorylated (p)-I $\mathrm{B} \alpha$ (cat. no. \#2859; used in western blotting) were purchased from Cell Signaling Technology, Inc. The antibodies against I $\mathrm{B} \alpha$ (cat. no. sc-371), P65 (cat. no. sc-372) and p-P65 (cat. no. sc-33020) were obtained from Santa Cruz Biotechnology, Inc., (Dallas, TX, USA) and were used in western blotting. The antibody against myeloperoxidase (MPO; cat. no. abs120616; used in IHC) was purchased from Absin Bioscience Inc. The second antibody (cat. no. 926-32211; used in western blotting) was purchased from LI-COR Biosciences.

Statistical analysis. All experiments were repeated at least three times independently. Continuous data were expressed as mean \pm standard deviation. Difference between groups were statistically analyzed using one-way analysis of variance followed by Student-Newman-Keuls' method with SPSS software version 19.0 (IBM Corp., Armonk, NY, USA). P<0.05 was considered to indicate a statistically significant difference.

\section{Results}

Chrysin pretreatment attenuates $I R$-induced renal dysfunction. To investigate the effect of chrysin on renal functions in renal IR injury, the chrysin pretreatment mice were injected with chrysin (Fig. 1A) for 3 days $(100 \mathrm{mg} / \mathrm{kg}$ each time) prior to the renal IR operation. The serum levels of BUN and $\mathrm{Cr}$ were significantly increased in the IR group compared with the sham group $(\mathrm{P}<0.05)$. However, chrysin pretreatment significantly decreased the levels of BUN and $\mathrm{Cr}$ $(\mathrm{P}<0.05$; Fig. $1 \mathrm{~B}$ and $\mathrm{C})$. These results indicate that Chrysin pretreatment attenuates IR-induced renal dysfunction.

Chrysin pretreatment attenuates renal IR-induced morphological abnormalities. Following renal IR, more severe tubular damage was observed in the IR group compared with the sham group. However, the abnormalities and tubular injury were significantly attenuated in the chrysin pretreatment group compared with in the IR group ( $\mathrm{P}<0.05$; Fig. 2A and B). These results indicate that Chrysin pretreatment attenuates renal IR-induced morphological abnormalities.

Chrysin pretreatment attenuates renal IR-induced apoptosis of tubular cells. The results presented above demonstrate that chrysin administration attenuates renal tubular damage. To investigate the effect of chrysin on tubular cell apoptosis, TUNEL staining was performed. The number of TUNEL positive cells was significantly increased in the IR group compared with in the sham group $(\mathrm{P}<0.05)$. However, chrysin pretreatment significantly decreased the number of apoptosis cells compared with the IR group ( $\mathrm{P}<0.05$; Fig. $3 \mathrm{~A}$ and $\mathrm{B})$. Considering that cleaved caspase- 3 protein is a marker of cell apoptosis and the protein level of cleaved caspase-3 was investigated by immunohistochemistry (IHC). Results demonstrated that the protein level of cleaved caspase-3 was significantly increased following renal IR injury $(\mathrm{P}<0.05)$. However, the expression of cleaved caspase-3 was significantly decreased in the IR+chrysin group compared with the IR group $(\mathrm{P}<0.05$; Fig. 3C and D). These results indicate that Chrysin pretreatment attenuates renal IR-induced apoptosis of tubular cells.

Chrysin pretreatment decreases the expression of Bax and cleaved caspase-3 and increases the expression of $\mathrm{Bcl}-2$ in renal IR injury. To detect the effect of chrysin on pro-apoptosis and anti-apoptosis associated proteins, a western blot assay was conducted (Fig. 4A). The protein levels of Bax and cleaved caspase-3 were increased and the expression of Bcl-2 was decreased in the IR group compared with the sham group. However, the protein levels of Bax and cleaved caspase-3 were significantly decreased, and the protein level of Bcl-2 was significantly increased in the chrysin pretreatment group compared in the IR group $(\mathrm{P}<0.05$; Fig. 4B-D). These results indicate that Chrysin pretreatment decreases the expression of Bax and cleaved caspase- 3 and increases the expression of Bcl-2 in renal IR injury.

Chrysin pretreatment attenuates renal IR-induced inflammatory response. To investigate the effect of chrysin on renal IR induced inflammatory response, the level of myeloperoxidase (MPO) by IHC was detected. The expression of MPO was significantly increased in the IR group compared with the sham 
A

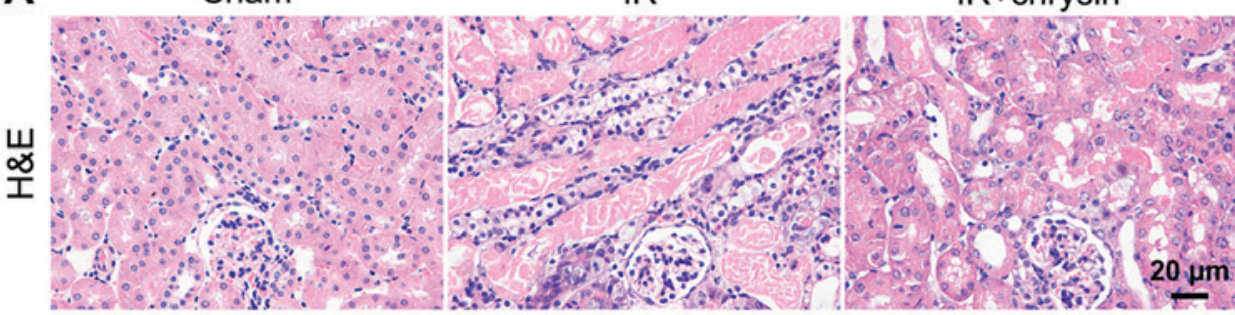

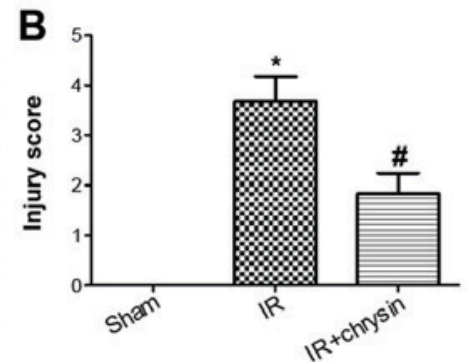

Figure 2. Chrysin pretreatment attenuates renal IR-induced morphological abnormalities. (A) The H\&E staining of sham, IR and IR+chrysin group. Scale bar, $20 \mu \mathrm{m}$. (B) Tubular injury score of the sham, IR and IR+chrysin group. For assessment of renal injury, tubular apoptosis, cellular casts and tubular injury were included. Score $0-4$ represent the injury area $<10 \%, 10-25 \%, 25-50 \%, 50-75 \%$ and $>75 \%$. ${ }^{*} \mathrm{P}<0.05$ vs. the sham group; ${ }^{\#} \mathrm{P}<0.05$ vs. the IR group. H\&E, hematoxylin and eosin; IR, ischemia reperfusion.

A

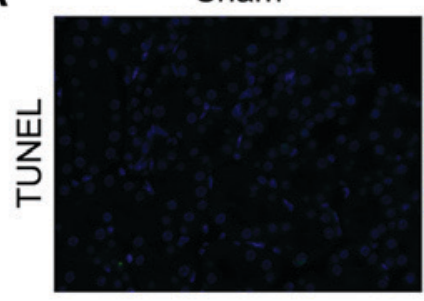

C

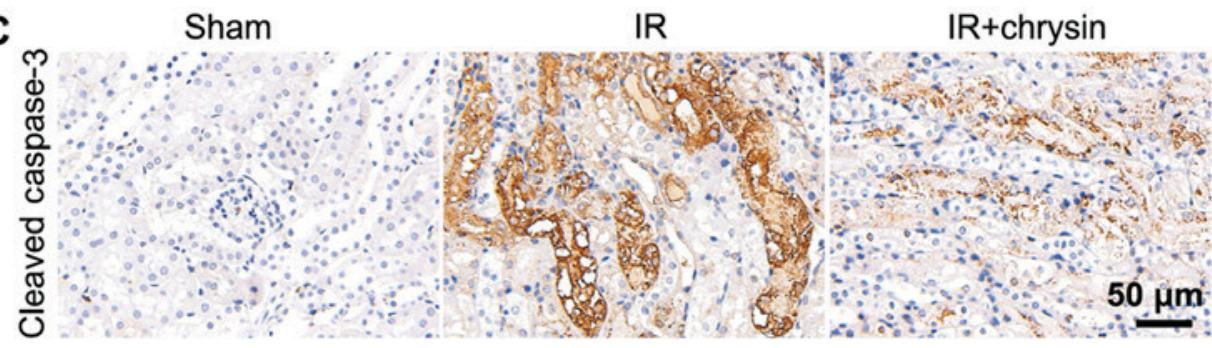

IR+chrysin
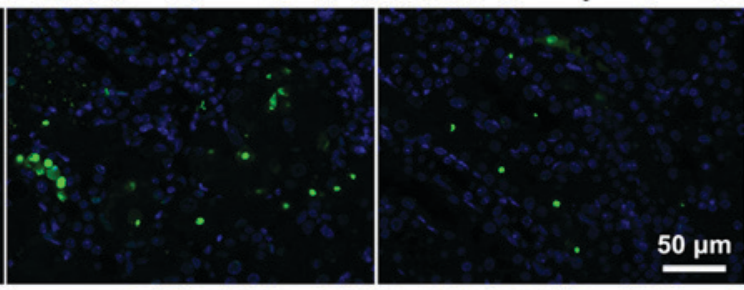

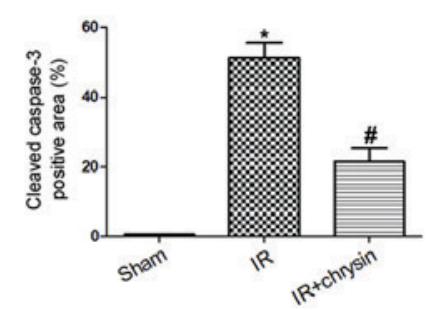

D

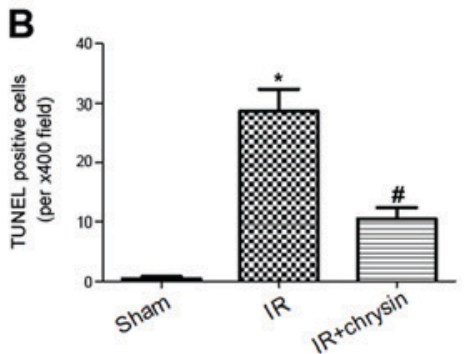

Figure 3. Chrysin pretreatment attenuates renal IR-induced apoptosis of tubular cells. (A) TUNEL staining of sham, IR and IR+chrysin group. Scale bar, $50 \mu \mathrm{m}$. (B) The number of TUNEL-positive cells of the sham, IR and IR+chrysin group were counted by Image-Pro Plus software version 6.0. (C) The immunohistochemistry staining of cleaved caspase-3 protein from the sham, IR and IR+chrysin group. Scale bar, $50 \mu \mathrm{m}$. (D) The positive areas of cleaved caspase-3 from the sham, IR and IR+chrysin group. ${ }^{*} \mathrm{P}<0.05$ vs. the sham group; ${ }^{\#} \mathrm{P}<0.05$ vs. the IR group. IR, ischemia reperfusion; TUNEL, terminal deoxynucleotidyl transferase dUTP nick-end labeling.

A

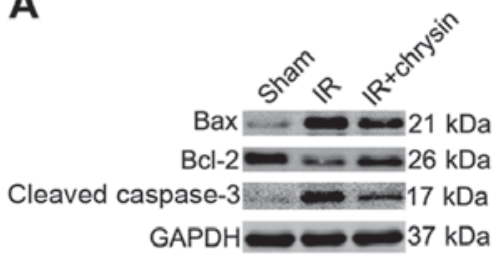

B

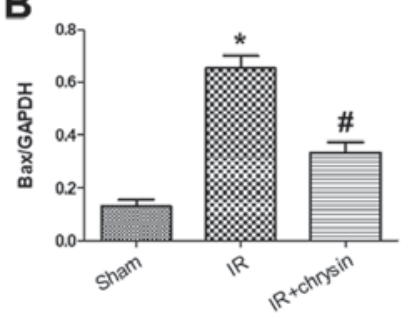

C

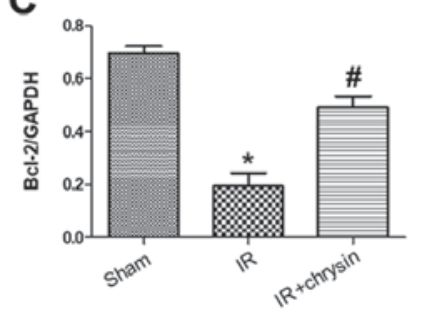




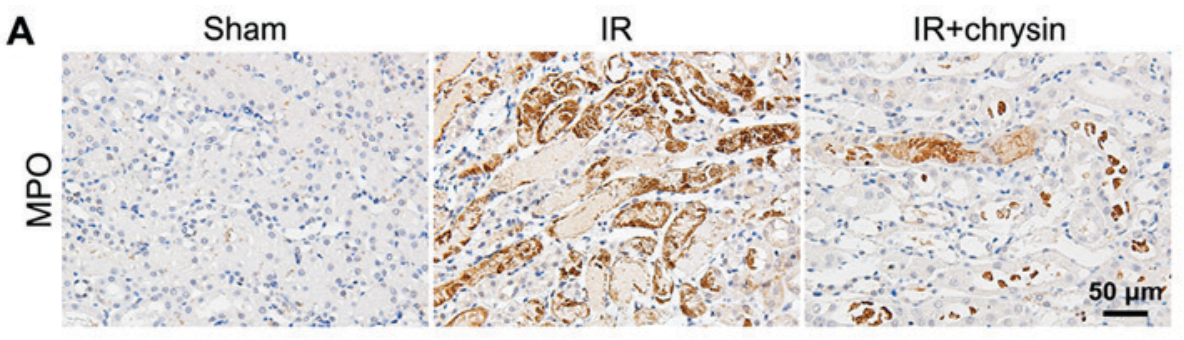

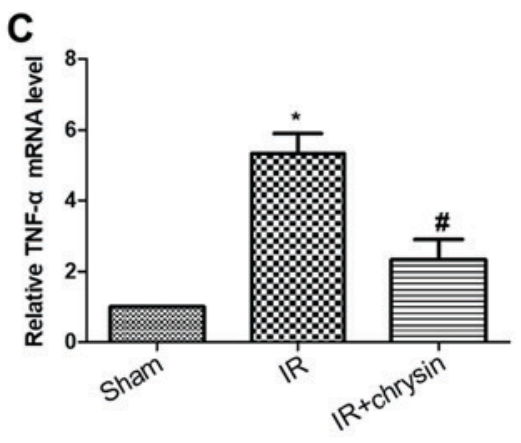

D
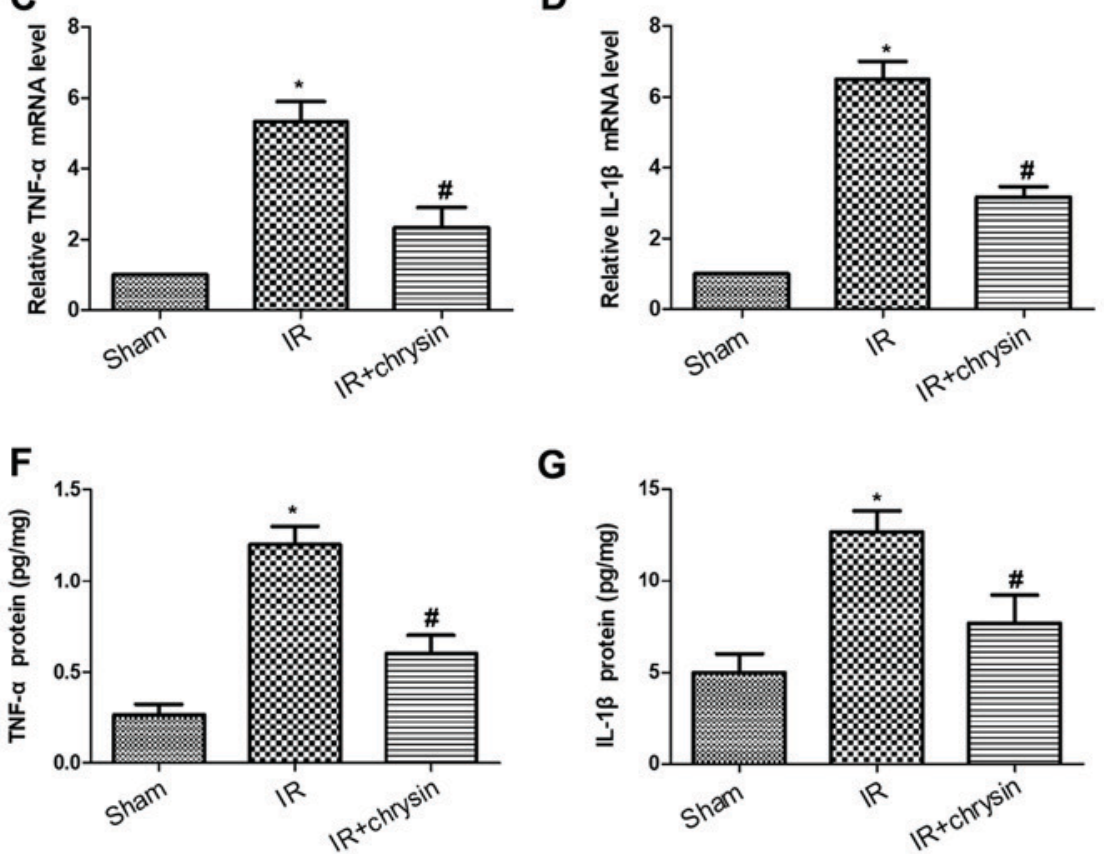

G

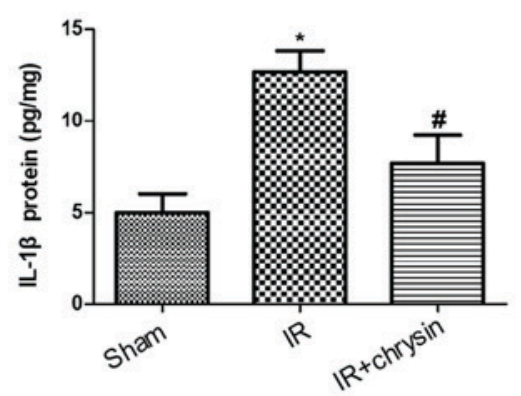

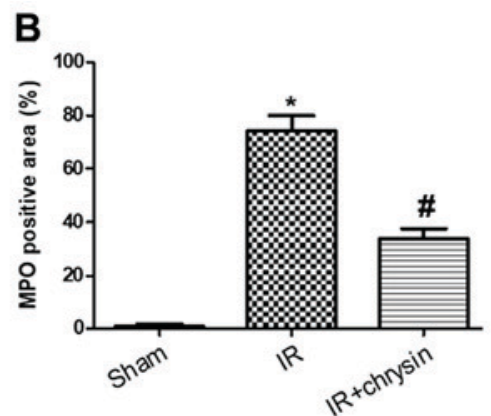

$\mathbf{E}$

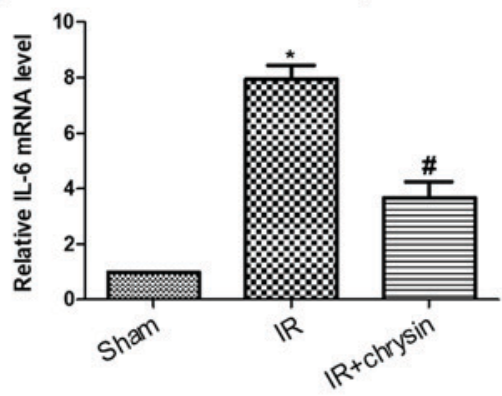

H

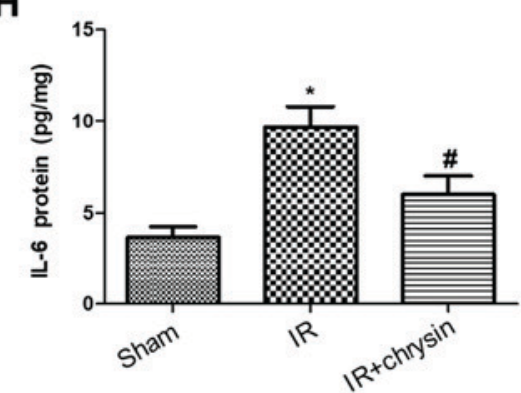

Figure 5. Chrysin pretreatment attenuates the renal IR-induced inflammatory response. (A) The immunohistochemistry staining of MPO in the sham, IR, and IR+chrysin group. Scale bar, $50 \mu \mathrm{m}$. (B) The assessment of MPO positive areas in the sham, IR and IR+chrysin group. The mRNA levels of (C) TNF- $\alpha$, (D) IL-1 $\beta$, and (E) IL-6 from the sham, IR, and IR+chrysin group by reverse transcription-quantitative polymerase chain reaction analysis. The serum protein levels of (F) TNF- $\alpha,(G)$ IL-1 $\beta$ and (H) IL- 6 from the sham, IR, and IR+chrysin group by ELISA analysis. "P<0.05 vs. the sham group; ${ }^{*} \mathrm{P}<0.05$ vs. the IR group. IR, ischemia reperfusion; IL, interleukin; TNF, tumor necrosis factor; MPO, myeloperoxidase.
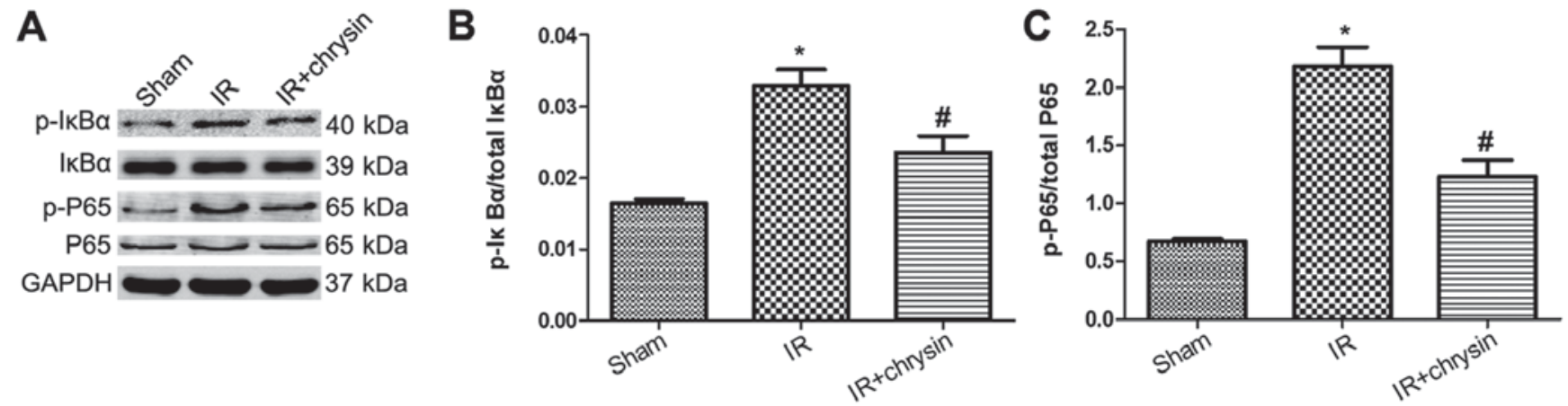

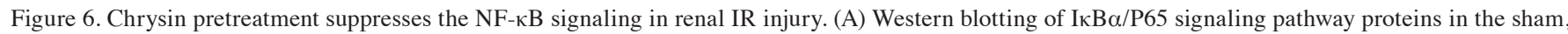

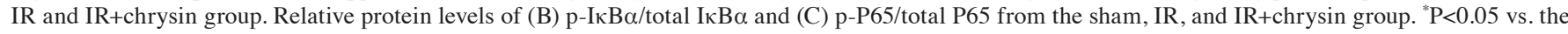
sham group; ${ }^{*} \mathrm{P}<0.05$ vs. the IR group. IR, ischemia reperfusion; NF, nuclear factor; p, phosphorylated.

Chrysin pretreatment suppresses the $N F-\kappa B$ signaling in renal IR injury. The NF- $\mathrm{BB}$ signaling is closely associated with renal IR injury (12). To investigate whether chrysin is involved in renal IR-induced NF- $\mathrm{KB}$ signaling activation, the protein levels of $\mathrm{I} \kappa \mathrm{B} \alpha / \mathrm{NF}-\kappa \mathrm{B}$ signaling were measured (Fig. 6A). The phosphorylation levels of I $\mathrm{B} \alpha \alpha$ and P65 were significantly increased in the IR group compared with the sham group $(\mathrm{P}<0.05)$. However, the phosphorylation levels of IкB $\alpha$ and P65 were significantly decreased in the chrysin pretreatment group compared with the IR group $(\mathrm{P}<0.05$; Fig. $6 \mathrm{~B}$ and $\mathrm{C})$. These results indicate that Chrysin pretreatment suppresses the NF- $\kappa B$ signaling in renal IR injury. 


\section{Discussion}

Ischemic AKI is a complex syndrome with multiple cellular abnormalities, resulting in accelerating cycles of tubular apoptosis, inflammation and renal injury (13). Clinically, no agent can effectively prevent renal injury following ischemia (14). In this study, the protective effects of chrysin on IR-induced renal dysfunctions and morphological abnormalities were reported. Furthermore, chrysin attenuated tubular cell apoptosis and inflammatory response in renal IR injury.

Chrysin is a bioflavonoid with anti-inflammatory $(15,16)$, anti-oxidant (17) and anti-carcinogenic (18) properties. In this study, a renal IR model was established in mice and pretreated with chrysin. Chrysin attenuated IR-induced renal dysfunction and morphological abnormalities. Chrysin particularly suppressed tubular apoptosis, inflammation and the I $\kappa \mathrm{B} \alpha / \mathrm{NF}-\kappa \mathrm{B}$ signaling pathway in renal IR injury.

Tubular cell apoptosis is a major pathogenic mechanism in ischemic AKI (19). The outer medulla suffers the most severe injury and apoptosis of tubular cells leading to renal dysfunction in renal IR injury (5). The levels of Bax and cleaved caspase-3 increase and the level of Bcl-2 decrease in renal IR injury, resulting in tubular cell apoptosis $(20,21)$. In the present study, the protein levels of Bax and cleaved caspase- 3 were decreased and the protein level of Bcl-2 was increased in the chrysin pretreatment group compared with the IR group. Consistent with these results, the number of TUNEL positive cells was decreased in the chrysin pretreatment group compared with the IR group. The results of the present study suggest that chrysin protects against renal tubular cell apoptosis induced by renal IR in mice.

The inflammatory response is another important part of the pathophysiology implicated in renal IR injury (22). Following renal IR, neutrophil infiltration markedly increases and pro-inflammatory cytokines, including TNF- $\alpha$, IL-6 and IL-1 $\beta$, are induced (23). In the present study, neutrophil infiltration and TNF- $\alpha$, IL-6, and IL- $1 \beta$ levels were decreased in the chrysin pretreatment mice compared with the IR group mice. The I $\kappa \mathrm{B} \alpha / \mathrm{NF}-\kappa \mathrm{B}$ signaling pathway serves an important role in the renal IR-induced inflammatory response (12). In the present study, the phosphorylation levels of $\mathrm{I} \kappa \mathrm{B} \alpha$ and P65 were increased in the IR group compared with the sham group. However, the phosphorylation levels of I $\mathrm{B} \alpha$ and P65 were significantly decreased in the chrysin pretreatment group compared with the IR group. Results suggest that chrysin functions as an anti-inflammatory agent in renal IR injury by suppressing the $\mathrm{I} \kappa \mathrm{B} \alpha / \mathrm{NF}-\kappa \mathrm{B}$ signaling pathway.

In conclusion, to the best of our knowledge this study provides the first evidence that chrysin attenuates renal dysfunction and morphological abnormalities in ischemic AKI. Furthermore, chrysin suppresses tubular cell apoptosis, inflammatory response and the NF- $\kappa$ B signaling pathway in renal IR injury. Therefore, chrysin may be a promising therapeutic agent for renal IR injury.

\section{Acknowledgements}

Not applicable.

\section{Funding}

No funding was received.

\section{Availability of data and materials}

The data and materials are available from the corresponding author on reasonable request.

\section{Authors' contributions}

DL designed the research. MX performed the experiments and wrote the manuscript. HS analyzed the data. All authors read and approved the manuscript.

\section{Ethics approval and consent to participate}

All experiments were approved by the Institutional Animal Care and Use Committee at Hubei University of Arts and Science. The surgical procedures were performed in accordance with the National Institutes of Health Guide for the Care and Use of Laboratory Animals.

\section{Patient consent for publication}

Not applicable.

\section{Competing interests}

The authors declare no conflict of interest.

\section{References}

1. Arai S, Kitada K, Yamazaki T, Takai R, Zhang X, Tsugawa Y, Sugisawa R, Matsumoto A, Mori M, Yoshihara Y, et al: Apoptosis inhibitor of macrophage protein enhances intraluminal debris clearance and ameliorates acute kidney injury in mice. Nat Med 22: 183-193, 2016.

2. Raup-Konsavage WM, Wang Y, Wang WW, Feliers D, Ruan H and Reeves WB: Neutrophil peptidyl arginine deiminase-4 has a pivotal role in ischemia/reperfusion-induced acute kidney injury. Kidney Int 93: 365-374, 2018.

3. Rabadi M, Kim M, D'Agati V and Lee HT: Peptidyl arginine deiminase-4-deficient mice are protected against kidney and liver injury after renal ischemia and reperfusion. Am J Physiol Renal Physiol 311: F437-F449, 2016.

4. Chen H, Wang L, Wang W, Cheng C, Zhang Y, Zhou Y, Wang C, Miao X, Wang J, Wang C, et al: ELABELA and an ELABELA fragment protect against AKI. J Am Soc Nephrol 28: 2694-2707, 2017.

5. Qin C, Xiao C, Su Y, Zheng H, Xu T, Lu J, Luo P and Zhang J: Tisp40 deficiency attenuates renal ischemia reperfusion injury induced apoptosis of tubular epithelial cells. Exp Cell Res 359: 138-144, 2017.

6. Yang L, Brooks CR, Xiao S, Sabbisetti V, Yeung MY, Hsiao LL, Ichimura T, Kuchroo V and Bonventre JV: KIM-1-mediated phagocytosis reduces acute injury to the kidney. J Clin Invest 125: 1620-1636, 2015.

7. Deldar Y, Pilehvar-Soltanahmadi Y, Dadashpour M, Montazer Saheb S, Rahmati-Yamchi M and Zarghami N: An in vitro examination of the antioxidant, cytoprotective and anti-inflammatory properties of chrysin-loaded nanofibrous mats for potential wound healing applications. Artif Cells Nanomed Biotechnol 46: 706-716, 2018.

8. George MY, Esmat A, Tadros MG and El-Demerdash E: In vivo cellular and molecular gastroprotective mechanisms of chrysin; Emphasis on oxidative stress, inflammation and angiogenesis. Eur J Pharmacol 818: 486-498, 2018.

9. Sassi A, Maatouk M, El GD, Bzéouich IM, Abdelkefi-Ben HS, Jemni-Yacoub S, Ghedira K and Chekir-Ghedira L: Chrysin, a natural and biologically active flavonoid suppresses tumor growth of mouse B16F10 melanoma cells: In vitro and in vivo study. Chem Biol Interact 283: 10-19, 2018. 
10. Yao Y, Chen L, Xiao J, Wang C, Jiang W, Zhang R and Hao J: Chrysin protects against focal cerebral ischemia/reperfusion injury in mice through attenuation of oxidative stress and inflammation. Int J Mol Sci 15: 20913-20926, 2014.

11. Livak KJ and Schmittgen TD: Analysis of relative gene expression data using real-time quantitative PCR and the 2(-Delta Delta C(T)) method. Methods 25: 402-408, 2001.

12. Jia Y, Zhao J, Liu M, Li B, Song Y, Li Y, Wen A and Shi L: Brazilin exerts protective effects against renal ischemia-reperfusion injury by inhibiting the $\mathrm{NF}-\kappa \mathrm{B}$ signaling pathway. Int $\mathrm{J}$ Mol Med 38: 210-216, 2016.

13. Dominguez JH, Liu Y, Gao H, Dominguez JN, Xie D and Kelly KJ: Renal tubular cell-derived extracellular vesicles accelerate the recovery of established renal ischemia reperfusion injury. J Am Soc Nephrol 28: 3533-3544, 2017.

14. Yuan X, Wang X, Chen C, Zhou J and Han M: Bone mesenchymal stem cells ameliorate ischemia/reperfusion-induced damage in renal epithelial cells via microRNA-223. Stem Cell Res Ther 8: 146, 2017.

15. Ramirez-Espinosa JJ, Saldana-Rios J, Garcia-Jimenez S, Villalobos-Molina R, Avila-VillarrealG,Rodriguez-Ocampo AN, Bernal-Fernandez G and Estrada-Soto S: Chrysin induces antidiabetic, antidyslipidemic and anti-inflammatory effects in athymic nude diabetic mice. Molecules 23: 67, 2017.

16. Choi JK, Jang YH, Lee S, Lee SR, Choi YA, Jin M, Choi JH, Park JH, Park PH, Choi $\mathrm{H}$, et al: Chrysin attenuates atopic dermatitis by suppressing inflammation of keratinocytes. Food Chem Toxicol 110: 142-150, 2017.

17. Missassi G, Dos Santos Borges C, de Lima J, Villela E Silva P, da Cunha Martins A Jr, Barbosa F Jr and De Grava Kempinas W: Chrysin administration protects against oxidative damage in varicocele-induced adult rats. Oxid Med Cell Longev 2017: 2172981, 2017
18. Lim W, Ryu S, Bazer FW, Kim SM and Song G: Chrysin attenuates progression of ovarian cancer cells by regulating signaling cascades and mitochondrial dysfunction. J Cell Physiol 233: 3129-3140, 2018

19. Zhou Y, Cai T, Xu J, Jiang L, Wu J, Sun Q, Zen K and Yang J: UCP2 attenuates apoptosis of tubular epithelial cells in renal ischemia-reperfusion injury. Am J Physiol Renal Physiol 313: F926-F937, 2017.

20. Choi DE, Jeong JY, Choi H, Chang YK, Ahn MS, Ham YR, Na KR and Lee KW: ERK phosphorylation plays an important role in the protection afforded by hypothermia against renal ischemia-reperfusion injury. Surgery 161: 444-452, 2017.

21. Wang Y, Mu Y, Zhou X, Ji H, Gao X, Cai WW, Guan Q and $\mathrm{Xu}$ T: SIRT2-mediated FOXO3a deacetylation drives its nuclear translocation triggering FasL-induced cell apoptosis during renal ischemia reperfusion. Apoptosis 22: 519-530, 2017.

22. Zhang Y, Hu F, Wen J, Wei X, Zeng Y, Sun Y, Luo S and Sun L: Effects of sevoflurane on NF-KB and TNF- $\alpha$ expression in renal ischemia-reperfusion diabetic rats. Inflamm Res 66: 901-910, 2017.

23. Raup-Konsavage WM, Gao T, Cooper TK, Morris SJ Jr, Reeves WB and Awad AS: Arginase-2 mediates renal ischemia-reperfusion injury. Am J Physiol Renal Physiol 313: F522-F534, 2017.

(c) () $\ominus$ This work is licensed under a Creative Commons International (CC BY-NC-ND 4.0) License. 